\title{
COVID-19 and mental health: A narrative review of relevant literature
}

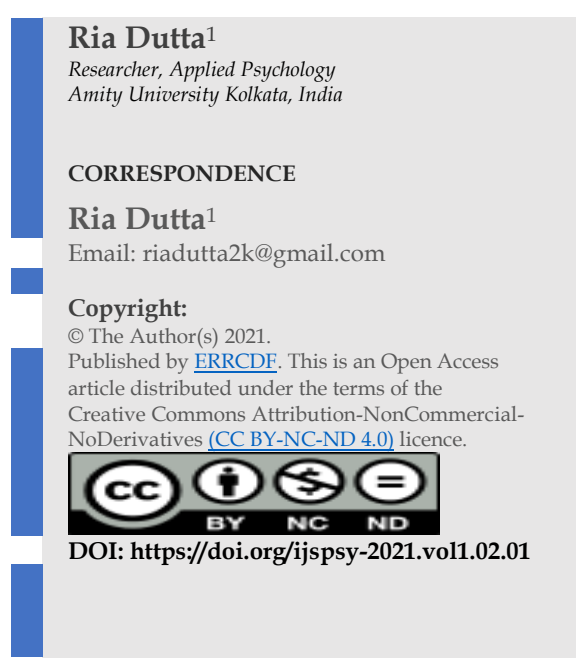

\begin{abstract}
In the late 2019 and early 2020s, a new variant of SaRs-CoV, now known as Corona virus or COVID-19, debuted internationally. Within a short time, the virus spread on such a scale that it resulted in drastic measures, like worldwide lockdown and strict social distancing, to fight against COVID-19. This study aims to explore the effects of COVID-19 and such protective measures on individuals from different population groups, ages, and walks of life. The literature review method was employed for this study where search words such as 'aged people', 'children', 'COVID-19', 'families', 'healthcare workers', 'mental health', 'psychological', 'social' and 'students.' were used. The findings of this study suggest that the psychological, emotional, and social complications of COVID-19 are not easily or quickly visible as the physical ones. After months of the COVID-19 pandemic, the mental health effects are starting to emerge and pose a serious threat to the well-being of those concerned and the broader society. Likewise, these mental health effects will not vanish as easily and are
\end{abstract} estimated to stay behind long after the COVID-19 pandemic is over. Anxiety, depression, compulsiveobsessive behaviour, hoarding behaviour, panic, stigmatization, and stress are some of the most common identified themes across different population groups. The need for mental health awareness and intervention strategies is ever so important now. Certain recommendations are also suggested in this paper.

Keywords: Aged people, Children, COVID-19, Families, Healthcare Workers, Mental Health, Psychological, Social.

\section{Introduction}

Our world so far has seen the emergence and extinction of different life menacing diseases. When the outreach of certain diseases exceeds nations' specific boundaries, it comes to be known with a new title, i.e. "Pandemic". A pandemic has time and again proved to be capable of jeopardizing the functioning of all societies and nations worldwide. In the past, humankind has witnessed the wrath of H1N1, SARS, Ebola, HIV, and the Spanish Flu (Manjunath, 2020), and the like. The year 2020 brings the new nation paralyzing disease known as Corona Virus 19 or better known as COVID-19.

In December of 2019, several cases came rushing into the hospitals of China with the same symptoms. By 12th January, the World Health Organization (WHO) identified the emergence of COVID-19 and warned against its unprecedented lethality and infectivity rates (Guo et al., 2020). As COVID-19 soon began to spread from region to region and from countries to countries at large, the countries started going into lockdown mode one by one and thus resulting in a worldwide lockdown and the strict practicing of social distancing and self-quarantine. On 24th March 2020, Indian Prime Minister Narendra Modi initially announced 21 days of nationwide lockdown (Hebbar, 2020). However, more extensions of lockdown were announced as the number of COVID19 positive cases, and several deaths kept rising. With the entire nation being put on hold, the tremendous impact of this can be seen in the business and organizational sectors and on an individual's and collective society's mental health.

With the workplace and education sectors turning to technology, graduation of students being delayed or virtual graduations being held, exams being delayed or cancelled, forbidden or restricted socialization, and dire need for wearing masks and maintaining proper hygiene being the reality, the need for exploring the effect of these on one's mental health became ever so important. Since its international debut, researchers and scientists from bioscience fields have extensively studied the physical effect of COVID-19, whose symptoms were then deemed to be visible within 11 days. However, the mental health effects often go unnoticed, undiscovered, 
neglected and untreated. Additionally, these mental health effects may not be as easy as the physical health effects to mitigate or cure. Therefore, there is an urgent need to study the mental health effects of COVID-19 and come up with action plans and intervention strategies.

\section{Objective of the Study}

This study aims to explore the psychological, social, and emotional impact of COVID-19 (from its emergence to its progression) on individuals from different age groups and different walks of life.

\section{Methodology}

A literature review is a foundation for this research study. Through the literature review method, research gets appropriate and relevant knowledge about the similar work done by various authors exploring the same domains (Ferenhod \& Fernandes, 2016). Through literature review, 30 research papers were collected. The search for relevant research papers was done through Google Scholar, PubMed, ScienceDirect, SAGE journals, Wiley Online Library, PLOS ONE, Indian Journal of Psychiatry website, World Social Psychiatry website, Cambridge University Press, IEEE, and Taylor \& Francis Online. The key terms used were: 'aged people', 'children', 'COVID-19', 'families', 'healthcare workers', 'mental health', 'psychological', 'social' and 'students.' Information from relevant newspapers was also collected from through Times of India, The Economic Times, The Hindustan Times and The Hindu. The search year for gathering information about COVID-19 was limited to the year 2020 (from January till October).

\section{Literature review}

Apart from the obvious and immediate biological effect of COVID-19, research throughout 2020 has explored the effect of COVID-19 in terms of psychological and social aspects of individuals. Social aspects of COVID-19 refer to the impact of COVID-19 on an individual's social sphere that includes the realm of one's social life, social relations, interpersonal communication, face-to-face contact, and the like. Psychological aspects of COVID-19 refer to the impact of COVID-19 that is more personal and on a more individual level.

\subsection{Psychological Aspects of COVID-19}

A study conducted by Moghanibashi-Mansourieh (2020) explored the level of anxiety among the Iranian population during the COVID-19 outbreak. The findings of this study suggest the anxietyprovoking nature of COVID19 in the Iranian population. Along with COVID19's infectious nature, the damage excessive anxiety and distress due to a body's immune system makes the individual all the more vulnerable to this menacing disease. An online questionnaire method was used where 10,574 respondents were collected and ultimately analyzed. This study showed that more than $94 \%$ of the participants "always/sometimes" follow the COVID19 news.

Consequently, the study shows that the more people follow the COVID-19 news, the higher their anxiety and severity levels. The level and the severity of anxiety symptoms of those residing in high prevalence regions were reported to be significantly higher than their counterparts, i.e. those who were residing in low prevalence regions. Age was also found to be an important factor for anxiety among the population. The group with the age range of 21- 40 years reported having significantly higher levels of anxiety than any other age group, gender, educational qualifications and prior direct (self) or indirect (family member) experiences of COVID19 were also prominent factors for predicting the level and severity of anxiety. The study concluded by reporting that age, location of residence, gender, educational qualifications, and prior experiences of COVID-19 were important for predicting anxiety in an individual.

A study by Huang and Zhao (2020) was conducted to assess and analyze the burden on the mental health of the Chinese public during the COVID-19 outbreak and explore further the potentially influential factors for such burden on mental health. A web-based cross-sectional survey was conducted where three standardized questionnaires were administered to 7,236 participants of the Chinese public. The study shows a high prevalence of Generalized Anxiety Disorder (GAD) and poor sleep quality among the Chinese public because of the COVID-19 outbreak. Anxiety symptoms were more prevalent in the younger population (less than 35 years old) and those who spent too much time focusing on the outbreak itself. The study showed that people who spent more than 3 hours focusing on the outbreak were more likely to develop anxiety symptoms. There were 
no gender differences reported for intensity and prevalence of anxiety in the population. Nearly $1 / 5$ th of the participants had depressive symptoms and sleeping problems due to the uncertainty in the epidemic progression. The study reported that possible reasons for the mental problems could be attributed to the "hypochondriac concerns" and the intense fear that the epidemic is hard to control. Healthcare workers were also reported to be at a higher risk of displaying psychological issues. The study concludes by suggesting that there should be more surveillance and monitoring of the psychological consequences of the epidemic and establishing early targeted mental health interventions to reduce the severity of the epidemic.

A study by Özdin and Özdin (2020) assessed the different levels of depression, general anxiety, and health-specific anxiety of individuals from Turkish society during the COVID-19 pandemic and explored the factors behind these. An online questionnaire method was used where two standardized questionnaires were administered on 343 individuals who were above 18 years of age. The study reports that depression, health-specific anxiety, and general anxiety scores were significantly higher among those female participants who live in urban areas and whose relatives, family members, or friends had a previous history of psychiatric or chronic illnesses (including COVID-19). According to the analyses, living in an urban area was evaluated as a risk factor for depression and general anxiety. This can be explained by considering the probabilities of encountering the virus being higher in the urban areas than in rural areas.

Additionally, women with past or present experiences with psychiatric illnesses or chronic illnesses have greater sensitivity and awareness of sensations of their bodies. Finally, this paper states that high levels of anxiety may adversely affect individuals' failure to comply with protective measures, thus harming their physical well-being. Therefore, the study concludes that psychological support measures for individuals from these groups should also be given the utmost importance in this health crisis.

A study by Roy et al. (2020) was conducted to understand the mental health issues emerging during the COVID-19 pandemic and the receptive strategies invented in the mental healthcare sector in the Indian context. A literature review was done, including collecting information from relevant research articles, newspapers, and websites to analyze the issues above and the possible underlying factors behind them. The study reports that the global mental health issues that emerged during the COVID-19 pandemic situation are depression, anxiety, stress, insomnia, denial, anger, fear, and even suicide (attempted/committed). There is an increased prevalence rate of mental health disorders among different population groups all over the world. This study analyzes the mental health implications for specific vulnerable population groups that include: (a) People with preexisting mental health illnesses; (b) Frontline workers; and (c) Children and older people. Some reasons for these mental health implications arise: social stigmatization, low priority to morbidities of mental health, little or inappropriate awareness of the virus and its risks, working under extreme situations, closure of schools, the shutdown of outdoor recreational activities, and social distancing. This paper concluded by discussing the mental health intervention strategies in both global and Indian contexts and further proposing a framework model based on this for future researches.

Imran, Zeshan, and Pervaiz (2020) conducted a study to understand the impact of COVID-19 on children, highlight their vulnerabilities to, and distress due to COVID-19, and propose intervention strategies that promote children's well-being and mental health in this COVID-19 pandemic situation. Relevant research articles and websites were referred to for collecting information. The study reports that children's response to an emergency like COVID-19 depends upon prior exposure to emergencies, physical and mental health, their families' socioeconomic factors, and their cultural background. In addition, this study highlighted that the COVID-19 pandemic has led to certain behavioural and emotional problems in children. Some of these problems are increased anxiety, excessive clinginess, irritability, fear that family members may contract the fatal disease, and increased screen time.

This article also reports that COVID-19 brings the risk of child abuse, child neglect, and child exploitation at home due to social distancing measures (Imran et al., 2020). Additionally, other population groups of children with special needs and children with pre-existing mental illnesses are particularly vulnerable to these negative psychological impacts of COVID-19. Shutting down of special schools or inability to receive proper psychiatric treatment may worsen the condition of 
such children. Furthermore, children in quarantine are also more likely to develop mental health disorders like anxiety and adjustment disorders. This study concluded that different and specific interventions could important for respective population groups of children to help parents safeguard their children's mental health in these challenging times. For example, younger children, older children, children with special needs, children under psychiatric care, and children in quarantine.

A study by Poudel and Subedi (2020) was conducted to study how COVID-19, lockdown, and practicing of social distancing have affected the overall physical, mental, social, and spiritual health of people. The literature review method was followed to gather relevant information from relevant articles and websites. The findings of this study report that lockdown and extreme social distancing are essential measures that help slow down or prevent the spread of COVID-19. The impacts on the following industries and sectors are observed: (a) Trade and tourism; (b) Education; (c) Agriculture; (d) Media sectors; (e) Health; (f) Vulnerable populations (e.g. migrants, minimum wage workers, people with disabilities). This study reports that being isolated and severely limited in social interactions have led to people being more distressed, anxious, and depressed. This has also led to certain behaviours like self-injury, substance use, discrimination, and even suicide. Media, instead of promoting individual well-being during these dire times, are compromising it. Media demand or expect people to develop a new skill during the lockdown, but not everyone can do that or want to do that in the first place. This creates a psychological burden and mental stress upon the individuals, provoking negative emotions like shame, guilt, and regret. Economic recessions have also taken a toll on the farmers and agriculture sector to the point that farmers commit suicide (Hossain et al., 2020). The study concludes by stating that the impact of COVID-19 on mental health is an indefinite challenge, and the authorities, like the government, must emphasize and address different coping strategies and mental health interventions to generate awareness and alleviate these negative effects on mental health.

Sheraton et al. (2020) studied the psychological issues such as anxiety, depression, occupational stress, PTSD, and insomnia of healthcare workers and non-healthcare workers during the COVID19 outbreak. A systematic literature review was followed where databases like Ovid, PubMed, Google Scholar, and Psych Info were searched for relevant research papers and articles by using specified keywords. These were "COVID 19", "Healthcare", "psychological," "SARS-CoV-2," and "Stress". The findings of this study suggest that there is no significant difference between the healthcare workers (HCWs) and non-healthcare workers (NHCWs) in terms of anxiety, depression, post-traumatic stress disorder (PTSD), and occupational stress. However, the psychological stressors for the two are different. Working with an infected patient was associated with occupational stress and anxiety for HCWs.

In contrast, uncertainties regarding the virus spread were associated with occupational stress and anxiety for NHCWs. The prevalence of insomnia was also reported to be higher in HCWs than in the NHCWs. This study also reports a risk of developing physical ailments such as headache, lethargy, and insomnia among the HCWs. The process of vicarious traumatization was also present not only in the HCWs but also in the NHCWs. The study concludes by stating that since HCWs and NHCWs are the ones who are responsible for handling the patients of COVID-19, there might arise some psychological issues as mentioned above that may affect their functioning and wellbeing. Thus, mental health services for the HCWs and NHCWs need to be considered. Such service should also be made an essential component in the healthcare system in the fight against any infectious disease.

Muruganandam et al. (2020) conducted a study on patients with severe mental illnesses (SMI) like schizophrenia, schizoaffective disorder, bipolar affective disorder, and major depressive disorder in the South Indian population. These patients were also reported to be from lower socioeconomic status (SES) backgrounds. The study aimed to understand the impact of COVID-19 on patients with SMI and identify its relationship with their knowledge of COVID-19. Telephonic-survey and questionnaire methods were employed for this study, and they were conducted on 132 patients diagnosed with SMI as per ICD (International Classification of Diseases) 10. The findings of the study report difference in the knowledge of COVID-19 among patients with SMI. This study suggests that $3 / 4$ th of the patients with SMI did not have adequate knowledge about COVID-19, 
its symptoms, and its protective measures to be taken. Some patients report not being aware of the ongoing COVID-19, its spread rate, and the lockdown situation; Some reported that they were only aware of COVID-19 due to someone else being screened positive; Some report being aware of its symptoms, and the majority of them did not report having any fear or worries related to contracting COVID-19.

Furthermore, this study reports that around $80 \%$ of the patients missed their appointments for their treatment of SMI in the previous months. $22 \%$ of the patients had to stop their psychiatric medications due to strict lockdown procedures or its consequences (pharmacy shops closed, lack of transportation, etc.) in Southern India. 3/4th of the patients were also reported to procure their medications by using their previous prescriptions. There were also reported instances of disturbed sleeping and eating patterns, re-emerging symptoms during the lockdown, suicidal ideation, and violence/aggression towards their caregivers. Financial troubles and caregiver stress were reported to worsen the situation. This study concludes by stating an urgent need for programs that target vulnerable populations, especially those belonging to low SES, low educational qualifications, and poor social support. Additionally, this study also advocates the management of patients with SMI to be put at the front in the future during any infectious disaster.

Dalal et al. (2020) published a research article that aimed to examine the emerging mental health issues observed during the COVID-19 situation and propose certain mental health strategies that can help tackle the issues in the current scenario for the ones to come in the future. The literature review method was employed where appropriate research articles and websites were consulted for adequate information. The research article reports that the COVID-19 pandemic situation provokes a lot of stress and uncertainty that can render individuals powerless and vulnerable to the development of mental health issues like anxiety, depression, and insomnia, resulting in poor individual well-being psychological distress.

At times, these mental health issues can facilitate severe physical illnesses like excessive anxiety and stress leading to heart attacks. Regardless of what kind of illness it is (mental or physical), certain actions should be taken to ensure these individuals are detected early and provided with appropriate care. This article shows what kind of mental issues and challenges are being faced by different population groups like (a) the general population; (b) psychiatric patients; (c) healthcare professionals; (d) office workers; (e) people at risk; and (f) special populations (children, pregnant women, the elderly, and the marginalized). The article concludes by stating specific intervention strategies that can help eliminate or reduce the mental distress being faced. Such strategies: (a) Telepsychiatry; (b) E-learning; (c) Teaching and practicing resilience. Finally, this article states that the emergence of COVID-19 has compelled the mental healthcare sector to reorganize itself to become more acceptable, accessible, and affordable.

Suryavanshi et al. (2020) conducted a study to assess the mental health about quality of life (QoL) of healthcare professionals (HCPs) during the COVID-19 pandemic. The online questionnaire method was used and administered on 197 healthcare professionals of India. The findings of this study suggest that there is a very high prevalence of depression and anxiety symptoms among HCPs. Single HCPs were found to be at a higher risk for experienced the combined effects of anxiety and depression when compared to their married counterparts. The reason for the experience of depression and anxiety symptoms was attributed to stressors related to the work environment (such as coming in contact with infected patients, overwhelming workload, lack of adequate knowledge and training, etc.). Moderate to severe depression and anxiety levels were independently associated with a poor or low Quality of Life. COVID-related stigmatization and social isolation can also emotionally disturb HCPs, leading to increased anxiety and depression. The study concludes by stating that HCPs are ever so important to the COVID-19 response and control efforts. Thus, there is an urgent need for rapid development and implementation of mental health interventions that prevent and treat the underlying mental health conditions of HCPs, both in India and internationally.

Roya et al. (2020) conducted a study to assess and understand the knowledge, attitude, anxiety experience, and perceived mental healthcare needs among the Indian population during the COVID-19 pandemic. The online questionnaire method was used and administered to 662 participants who were above the age of 18 years. The findings of this study suggest that the 
participants had moderate to high levels of awareness about the virus and its preventive measures. The reason attributed to this is that media and government sources have emphasized innumerable times about COVID-19 since March. Additionally, it is assumed that the participants having good educational backgrounds and professions related to healthcare gave them an added boost to be more aware of the ongoing situation. This awareness of COVID-19 and its risks is reflected in their behaviour to use sanitizers, handwashing, and masks frequently. They also practice appropriate social distancing, self-quarantining, avoid travel and maintain proper hygiene.

Awareness has also been associated with the reduction or absence of stigmatization of COVID-19. However, regardless of one's educational level or professional background, COVID-19 has also been reported to have other psychological effects on the participants' panic buying of supplies (hoarding), having sleep difficulties, being worried, and being anxious after reading or discussing any COVID-19 related topics. This also shows the importance of media in circulating information while also generating anxiety among people. Thus, media influences the well-being of citizens and adds to the level of anxiety. This study concludes by stating that no matter how educated and aware general people and health professionals are, there is still an increasing amount of worry and apprehension among the public regarding COVID-19. Thus, appropriate mental health interventions must be devised urgently.

Prentice, Zeidan, and Wang (2020) conducted a study to study how individuals' personality traits and emotional intelligence influence their coping strategies to COVID-19. Online questionnaire method was used, and 468 responses were collected. The respondents were Chinese residents aged 18 and above and understood the impact of COVID-19 and government interventions. The findings of this study suggest that although both male and female participants were task-oriented in terms of coping, female participants were more inclined to seek emotional support. In contrast, male participants were a more avoidant type or engaged in drinking or hedonistic behaviours. Female participants were also reported to be perceived more as nurturing or caring figures than the males. Older participants reported feeling more responsible for protecting their families.

Among the five personality factors, the openness to experience factor was significantly related to avoidance-oriented coping, task-oriented coping, and emotion-oriented coping strategies. It was also found to be negatively related to avoidance-oriented coping. The next personality factor, conscientiousness, was also found to be positive associated with emotion-oriented coping and negatively associated with avoidance-oriented coping strategies. Emotional Intelligence (EI) was also significantly related to task-oriented, emotion-oriented, and avoidance-oriented coping strategies. This study concluded by stating that personal factors (i.e. personality traits and EI) are important indicators of an individual's coping strategies and behaviours. However, the EI has the most significant impact on the individual's positive and proactive coping styles. Therefore, authorities are encouraged to incorporate individual traits in crisis management research to help shape their public policy making.

Gopal, Sharma, and Subramanyam (2020) conducted a study to explore the effects of extended lockdowns of COVID-19 on individuals. It was a longitudinal study that lasted from March till May. Online questionnaires were administered at each phase of this study. Only 159 Indian participants completed all the study phases; thus, only their responses were included for further analyses. The findings of this study suggest a statistically significant increase in stress, anxiety, and depression symptoms. This increase was found to be greater among women than in men. Individual's resilience was also found to be negatively correlated with anxiety, stress, and depression symptoms. This study suggests that the extension of lockdowns has harmed individual's psyche. The unpredictability or the perception of it can explain the elevated levels of anxiety.

In contrast, the financial losses combined with social isolation can explain stress levels and depression symptoms. The gender differences between the anxiety, stress, and depression symptoms were associated with gender roles and tasks at home. Additionally, this study also reports a relapse of psychiatric disorders for patients with a history of mental illness. This study concludes by stating that COVID-19 is not just a medical phenomenon and has psychosocial impacts on individuals. It encourages society to address these impacts that are causing psychological distress at large. 
Hao et al. (2020) conducted a study to assess and compare the psychological impact of COVID-19 after strict lockdown measures on individuals with and without psychiatric illnesses. Online questionnaire methods were administered on 109 healthy individuals aged 18 or above and never having a history of psychiatric illnesses and 76 psychiatric patients being officially diagnosed as per ICD 10's criteria. The findings of this study report that psychiatric patients scored significantly higher in anxiety, depression and stress subscales. They were also the ones who were significantly more likely to report their worries about their health, anger, impulsivity, suicidal ideation, and severe insomnia. The study further adds that during this COVID-19 and lockdown situation, patients with psychiatric illnesses were more likely to endure higher levels of PTSD as well. Psychiatric patients were also estimated to see a reduction in their mental health services. They were more unable to fulfil their mental health needs, resulting in higher relapses or worsening their current conditions. Psychiatric patients were also moved to a lower priority as the number of COVID-19 cases rose sharply. This study concludes by stating that the results obtained from this research can be used as a reference point for mental health professionals and authorities to identify potential targets of assessment and psychiatric care for such patients during the COVID-19 pandemic before it is too late.

Shuja et al. (2020) published a research article that aimed to understand and explore the mental health implications of the COVID-19 pandemic on an individual. This study followed the literature review method, where they gathered relevant information from relevant research papers. The findings of this study suggest that the rapid increase of COVID-19 cases and deaths, extreme lockdown measures, strict governmental policies, and harsh social distancing are taking a toll on the mental health of individuals. There is an increasing amount of psychological distress, panic, and anxiety in individuals who are (supposedly or actually) exposed to this virus all around the globe. The role of media is also reported to be crucial here. The media has underlined COVID-19 as an exclusive threat that has added panic and stress among the masses, which has led to the development of worsening of mental disorders like anxiety, depression, obsessive-compulsive disorder, and post-traumatic stress disorder. Aggression, violence, stigmatization, and social seclusion are also reported to impact the social aspect. This study concludes by stating that COVID19 has spread fear at individual levels and societal levels, and immediate mental health interventions must be taken.

\subsection{Social Aspects of COVID-19}

A study conducted by Dubey et al. (2020) explored the psychosocial impact of COVID-19 on healthcare workers, children, aged people, domestic caregivers, psychiatric patients, and the marginalized community (migrants, slum dwellers, and prisoners). The paper reviewed the existing literature on COVID-19 on several databases like PubMed and Google Scholar. The paper reports that COVID-19 has evolved to become stigmatized over the months. The term "corona positive" brings dreadful health news and the threat of stigmatization, which leads to fear of isolation, racism, discrimination, and marginalization within all the socioeconomic strata of society. To present and control the reach of the disease, quarantining and lockdowns have been implemented. Nationwide lockdown programs have been reported to produce mass hysteria, depression, anxiety, and tremendous levels of distress in both individual and collective levels. The study concludes by stating that these effects are intensified when coupled with financial losses, stigmatization, and receiving inappropriate or inadequate information about the disease from the traditional and digital media and the netizens.

Nicomedesa and Avila (2020) analyzed the nature of panic responses of the people and their perceptions of the current global issue of COVID-19. A mixed-method approach was followed for this study where the data of 538 participants (age ranging from 13-67 years) were analyzed quantitatively and then qualitatively. Quantitative data analysis reported "moderate levels" of Illness Anxiety for COVID-19 and reported certain hypochondriasis symptoms. The anxiety level was not too high to be seeking urgent psychological interventions, but it was neither too low to be neglected in the background. There were also significant differences between Illness Anxiety and locations concerning awareness levels, educational qualifications, and proximity to the COVID-19 cases associated with the locations. Qualitative analysis of the data done propounded the 'Panic Framework', which provides a spectrum of feelings, thinking patterns and behaviour of the 
participants caused by the COVID-19 pandemic. Ranging from negative end to the positive end, this spectrum includes Indifference, Annihilation, Nihilism, Paranoia, Sadness, Fear, Transmission of Virus, Shock, Government Blaming, Anxiety, Relating to Past Pandemics, Worry of Oneself/Family/Others, Information Dissemination, Composure, Compliance, Protection, Cautiousness, Optimism, and Health Consciousness. The study concluded by stating that the level of symptoms of hypochondriasis, attitude on acquiring COVID-19, avoidance behaviour, and reassurance seeking of the participants were at moderate levels.

A study by Kochhar et al. (2020) was conducted to explore and assess the impact of lockdown on the mental health of the population of New Delhi. A mixed-method approach was used here where along with the literature review, data of 992 participants (aged 21-65 years) was collected based on three questionnaires. The findings suggest that $98.2 \%$ of the participants were following the COVID-19 and social distancing guidelines. A positive correlation between the awareness of and practicing social distance or isolation was found. $78.5 \%$ of the participants were optimistic about overcoming this pandemic situation, and $12.1 \%$ felt helpless and depressed. Nearly half of the participants had reported having sleeping troubles due to high levels of anxiety and stress because of lockdown. $79.5 \%$ of the participants reported having changes in their eating habits, and $26.3 \%$ stated that they started consuming substances (e.g. alcohol, drugs, and tobacco) due to lockdown. Stressors relating to finances were also reported for most participants as the lockdowns kept getting extended. Fear of acquiring COVID-19 and being treated differently for it were also reported for the participants. This study concludes by proposing authorities take strategic actions and plan lockdowns to become more effective without compromising the mental health of the people.

Similarly, Serlachius, Badawy, and Thabrew (2020) explored the challenges and opportunities of youth with chronic health conditions during the COVID-19 pandemic that has impacted these youth and children's mental and physical well-being. Literature review methodology was adopted to gather relevant information about the topic. This article reports the challenges observed among the children and youth with chronic health conditions population groups. These challenges are heightened health anxiety, disrupted daily routines, academic and social difficulties arising out of the closure of schools and educational institutes, Family stress and increased risk of domestic violence, and overall reduced access to physical and psychosocial support. Although all these stressors are faced worldwide, the population groups for this study are particularly more vulnerable to these stressors. The paper concludes by stating the psychosocial interventions that can be taken to overcome the challenges above. These strategies include: (a) promoting less academic stress, (b) having and seizing the opportunity to spend more quality time with the family and understanding them; (c) building resilience; (d) accessing more healthcare technology; and (e) reducing (if not eliminating) the access to substances like tobacco, alcohol, etc.

Majumdar, Biswas, and Sahu (2020) studied the effects of the pandemic, social distancing, and stayat-home confinement on India's corporate sector professionals and undergraduate and postgraduate students. The questionnaire method was adopted to assess the sleep parameters, depressive symptomatology, and somatic discomfort of 203 individuals working in the corporate sector and 325 undergraduate and postgraduate students. The findings report an increase in the presence and intensity of health morbidities just before and during the lockdown phases. Office workers were reported to spend a larger significant amount of time on their computers than the students, while the students were reported to use cell phones more significantly than the office workers. Being confined to the home and having a sedentary lifestyle due to lockdowns was associated with bodily discomfort by both office workers and the students. Sleep duration and quality of life of the office workers were found to be disturbed significantly.

On the other hand, contrasting results were found for the students - that is, their sleep duration increased, and their sleep quality was also good. In addition, depressive symptomatology was found to have increased two-fold in the office workers' case, while the same was found to have increased three-fold for the students. The study concludes by stating that lockdown has had a significant effect on each of the domains above of students' and office workers' lives that can disrupt mental health and physical well-being if they are left unchecked. 
Chen, Rubens, and Li (2020) explored the acceptance of smart home technology (HEMS) and willingness to pay (WTP) for HEMS by the residents during the COVID-19 pandemic. Interviews were conducted with 623 participants residing in New York and who were aged between 30-69 years. The findings of this study show that the usage of electricity was consistent from mornings to evenings, and there were no usual peaks and falls that were present pre-COVID19 times. Consequently, it was reported experiencing paying much more for electricity than it did on average. Participants were also reluctant to adopt HEMS (smart home technology) for energy saving. Cybersecurity concern was positively related to the adoption of HEMS. Attitude perceived behavioural control for electricity usage and social norms was the strongest predictor of willingness to pay (WTP) and adoption of HEMS. Technology anxiety was also found to influence WTP for well-being features positively. Additionally, it was also found that participants who had a moderate or higher perceived risk of being infected by COVID-19 were willing to pay more than their counterparts. This study concludes by stating that this study can help understand the multidimensional impact of COVID-19 concerning technological and social attributes.

A study by Khattar, Jain, and Quadri (2020) was conducted to explore and understand the day-today living activities and learning styles of young students for their mental health. An online questionnaire method was used where the responses of 583 young Indian students were collected. These findings of the study report that lockdown has shifted every student to do most of their academic and recreational activities online. Turning to virtuality would seem like a good solution to the problem. However, while nearly half of the participants reported having unlimited access to the internet, the other half faced consistent bandwidth problems. The number of hours these students spent in front of a screen was reported to range from more than 12 hours a day to 30 minutes a day.

For recreational activities, the participants reported spending more than four hours a day on social media. WhatsApp was reported to be the most popular application, to be used amongst students for communications within a closed group, which was followed by Instagram and YouTube. The majority of the participants reported spending more time with their families. Internet gaming, watching movies, sleeping and cooking were reported to be the top four recreational activities reported by the students. From the analysis of the data, it was seen that nearly more than half of the participants miss seeing their schools, colleges, and friends personally.

Additionally, nearly only $20 \%$ of the participants reported feeling connected as a group while attending their online classes and that online classes are helpful. Although participants have adapted well to stay at home and to using e-learning, they also experience anxiety and major learning disruptions as their prime concerns were about examinations and admissions to the next level of education. This study concludes by stating that factors such as less physical activity, prolonged screen time, monotony, inappropriate sleeping and eating patterns, frustrations, network problems, etc. can have detrimental effects on these young students' minds.

Mukherjee, Bandopadhyay, and Chatterjee (2020) published a research article that aimed to explore the effects of the COVID-19 pandemic on mental health and beyond from an Indian perspective. The literature review method was followed where relevant information from other research articles and websites was taken. This research article reports that early lockdowns had helped tremendously in somewhat slowing down the infectivity rate of COVID-19 in India. Still, it compromised the functioning of every sector in the society. Now the question of dilemma that arises is whether to lift the lockdown or not. Following the Kerela model, this article reports that the authorities should ensure a high number of rigorous testing per day to identify both symptomatic and asymptomatic carriers of COVID-19 and take necessary actions immediately. The negative effects of prolonged lockdowns are seen in the social and economic areas where unemployment rates and turnover rates are rising, and companies are going out of business. A huge portion of the population who live in the underprivileged threshold is dying of hunger and living in fear of when this nightmare will be over. For these marginalized population groups, social distancing is a luxury.

The psychological impacts on these population groups are estimated to be huge. The article also reports that there's an increasing amount of stigmatization is associated with acquiring COVID-19. A COVID-19 positive patient is looked down upon and even outcasted. This can result in people 
hiding or distorting their identity and test results and being reluctant to seek medical help, leading to the worsening of the COVID-19 pandemic situation. Additionally, this article also states that anxiety, fear, stress, and panic are the most predominant manifestations, while irritability, anger, aggression and psychosis are other externalising behavioural manifestations of COVID-19. The research article concludes by stating that India is not new to epidemics and pandemics, and although it has jolted the nation, it too shall pass if effective measures are taken at the individual and governmental level.

Chaturvedi and Sharma (2020) published a research article that aimed to examine and understand the psychosocial aspects and effects of COVID-19 from an Indian perspective. Literature review method was used where they gathered relevant research articles and websites for appropriate information. This research article reports that there's been an increasing rise in the negative social trend of stigma associated with COVID-19 that has affected a large variety of sections of society. This stigma leads to social issues like discrimination, isolation, seclusion and even violence. COVID-19 has also led to emotional disturbances and maladjustments within families, and especially within the families of persons with mental illnesses. Negative expressed emotions have worsened that enhance the risk of aggravation of the mental health issues and risk of relapses. With the phenomenon of working from home and attending classes from home, the concept of a traditional "home" has also been disturbed by this lockdown. Social distancing and home quarantine have also been assumed to worsen one's social interaction skills and/or stagger the further development of such skills. In other words, because of the absence of non-verbal communications, we are all missing out on a very important aspect of social development and communication. There has also been a rise of reported domestic violence in dysfunctional homes but there is no other available escape from this dysfunctional home environment because of lockdown. This research article concludes by stating that COVID-19 does not only impact one's physical health but also one's psychosocial development. It further proposes that instead of using handshake for greeting, we can use "Namaste" for greetings to minimalize touching another human being while also making sure to show them acknowledgement or respect.

Bhattacharya, Banerjee and Rao (2020) published a research article that seeks to examine and highlight the effects of social stigma and its consequences considering COVID-19 situation. They employed the method of literature review for their article and gathered relevant information from other research papers and websites. This research article reports that COVID-19 has generated social stigmatization and encouraged discriminatory behaviour towards individuals have COVID19 or are perceived to have contact with it. It is reported that COVID-19 has a biological impact and consequences but the psychosocial consequences will persist longer than the biological ones. This social stigmatization has strained both inter-regional and international relations. Stigmatization is not just limited to the victims of COVID-19 but also to the healthcare workers, nurses, frontlines workers, police personnel, etc. All these lead to individuals hiding their test results in order to not be discriminated. This leads to the worsening of the COVID-19 situation and of the individual's health as well. This article concludes by stating that to mitigate or eliminate social stigmatization, medias should frame their content with sensitivity, and us netizens should all act responsibly, in a manner that reflects a fight against the disease and not a fight against the person with the disease.

Wardell et al. (2020) conducted a study to examine the coping-motivated drinking patterns leading to alcohol use and alcohol problems during the COVID-19 pandemic. Semi-structured interviews and questionnaire methods were employed on 320 Canadian participants. The findings of this study suggest that COVID-19 pandemic has led to drastic changes in one's daily functioning. These changes are associated with elevated levels of distress, anxiety and depression. The findings report that individuals who have a child under the age of 18, having depression and having lower social connectedness predicted unique variance for coping motives that in turn predicted increased alcohol use. Therefore, the study concludes by suggesting that coping motives were associated with increased alcohol consumption and this, in turn, was associated with greater alcohol problems. Additionally, income loss and living alone was associated with increased alcohol consumption and solitary drinking, but they were not associated with coping motives. This could mean that observed solitary drinking during pandemic is more associated with situational factors 
rather than coping processes - maybe these individuals drank outside with their friends but due to lockdown, they drink alone at their homes by themselves.

Rajkumar (2020) conducted a study that aimed to explore the effect of COVID-19 on mental health. Systematic literature review method was used. With the help of set and specified keywords, relevant research papers were gathered from only the database of Pubmed. The findings of this study suggest that COVID-19 pandemic is multidimensional in nature, affecting one's physical well-being, social well-being and psychological well-being, and thus it requires a multifaceted response from psychiatrists and allied professionals so that the overall mental health can be holistically taken care of for both the general and the vulnerable populations. The long-term impact of COVID-19 on mental health may take months or years to become fully apparent and managing this impact requires efforts from not only psychiatrists but also the healthcare system. COVID-19 pandemic situation has given rise to both challenges and opportunities for making improvements in the mental health sector. This study concludes by stating that there is an urgent need to develop mental health interventions that are time-limited, culturally sensitive and can be taught to all the healthcare workers and volunteers.

Collado-Boira et al. (2020) conducted a study to understand the perceptions and fears of medical students due to their immediate voluntary incorporation in professional practice by the government for COVID-19 measure. A phenomenological approach was followed with the help of semi-structured interview and questionnaires administered on 147 Spanish medical students. This study reports that Healthcare workers have been proved as the frontlines of COVID-19 pandemic. COVID-19 revealed a significant lack of nursing staff in the medical sectors. The Spanish government requested immediate voluntary participation of medical students for COVID-19 measures. Data analysis suggests that one of the main fears these medical students experienced was the fear of getting infected - they were concerned for their safety, but they were also willing to work despite the potential risk of infection.

Another factor that was identified among the Spanish participants, besides concern for personal health was the fear of infecting their significant others, family members or relatives. Another factor that was identified among the medical students was related to the perception of professional training and competency for the COVID-19 works they were assigned to. Participants reported feeling the lack of professional knowledge and hand on skills, which made them feel incompetent, inferior and unfit for such an important job. The last factor that was identified was coping with patients' deaths. Being only medical students and lacking professional expertise or in-field experience, they felt they were not ready to deal with patient deaths. This study concluded by stating that lack of professional skills and professional experience, coping with difficult situations and fear of virus transmission were major student concerns that should be taken into consideration to also ensure the overall well-being of healthcare workers.

Kontoangelos, Economou and Papageorgiou (2020) published a research article that aimed to explore the mental health effects of COVID-19 in the general population. The literature review method was used where they reviewed 65 papers across the database of Medline. The findings of this study suggest that children and aged people are more likely to experience worry, anxiety symptoms and fear. The COVID-19 pandemic has been reported to be extremely fear-inducing or extremely frightening due to its very nature of infectivity, lethality and unpredictability. Due to strict lockdown measures taken by the government, the general public and the special populations are both under physical, financial, social and emotional pressure. This study concludes by stating that constructive peer-support, supportive therapy, early mental health interventions, telepsychiatry, controlled media information circulation, etc should be prioritized and practiced globally.

A study by Grover et al. (2020) was conducted to evaluate the psychological and social impact of COVID-19 on the general population of India by assessing the prevalence of depression, anxiety, perceived stress, well-being and other psychological issues. Online survey was conducted where four standardized questionnaires were administered on 1871 participants but only the responses of 1685 participants were analyzed because the remaining participants' responses were incomplete and were, thus, omitted. The findings of this study reported that $70 \%$ of the participants reported moderate levels of stress after the onset of the lockdown period; Nearly $50 \%$ of the participants 
reported better improvements in their relationships with their partner, children, parents, neighbours and colleagues after the beginning of the lockdown period; $30.7 \%-36 \%$ of the participants reported mild to severe worsening of negative emotions (e.g. sadness, anxiety, irritability, frustration, and fear); $18.7 \%$ of the participants reported marked increase in social media usage; $18.3 \%-31.7 \%$ of the participants reported slight to moderate increase in activities such as exercising, worshipping God, watching movies, gaming, sexual activities, etc. Majority of the participants reported having the fear of getting infected with COVID-19. This study concludes by stating that while there is a prevalence of negative emotions about the virus and lockdown, majority of the participants reported having a positive impact of lockdown in the social relationship dimension. The study attributes this to the fact that everyone realized that they are fighting a common enemy in this global health crisis. Thus, interpersonal relationship issues are put on the backburner, for now, reflected in marked improvements in relationships.

\section{Discussion}

People do not always feel happy and cheerful. They get sad, unhappy, angry, and unwell too. Despite that, mental health has been conceptualized as having overcome the aforementioned negative affect and having a positive affect that is marked by the feelings of happiness and having a marked sense of mastery over one's environment (Lamers et al., 2011). Keyes (2014) has identified three aspects of mental health. They include Emotional well-being, Psychological well-being, and Social well-being. Based on the overall understanding of the concept of mental health, the finding of this paper analyzes the complications of COVID-19 under the following three headings: (a) Emotional complications; (b) Psychological complications; and (c) Social complications.

\subsection{Emotional Complications}

Ever since the first time of making the news headlines by the novel SARS-CoV (i.e. Corona Virus19), it has sparked feelings of anxiety and intense fear among the masses worldwide. To prevent or control the spread of infectivity of this virus, governments worldwide have initiated strict lockdown measures and the practice of social distancing. Unfortunately, this has only added fuel to the fire - it has boosted the fears and concerns of the masses. Several studies throughout 2020 have reported the relationship between COVID19 and mental health for different population groups. These groups include (a) The general population, (b) Children and the elderly population, (c) Frontline and non-frontline workers of COVID-19; (d) Individuals with pre-existing psychiatric illnesses; (e) Individuals with special needs; (f) Marginalized population (migrants, wage workers, and prisoners) (Dalal, et al., 2020; Imran, Zeshan \& Pervaiz, 2020; Muruganandam, et al., 2020; Roy, et al., 2020; Sheraton et al., 2020).

\section{Anxiety}

Ever since the outbreak of COVID-19 in China, studies have shown an increased prevalence of Generalized Anxiety Disorder in the general population (Huang \& Zhao, 2020). Researches show that although everyone feels anxious about the pandemic, the severity and intensity of anxiety depends with age, gender, location of residence, educational qualifications, prior experiences of COVID-19 and how much time the individual spends following the updates of COVID-19 (Huang \& Zhao, 2020; Moghanibashi-Mansourieh, 2020; Özdin \& Özdin, 2020). Apart from general anxiety, health specific anxiety or hypochondriac concerns were also reported (Huang \& Zhao, 2020; Özdin \& Özdin, 2020). Some amount of anxiety can be healthy as anxiety can alert an individual about the potential risks of the disease, however, anxiety beyond the normal threshold can be detrimental to the health and immune system (Moghanibashi-Mansourieh). Additionally, high levels of anxiety can adversely affect the individual's ability to comply with protective measures (Özdin \& Özdin, 2020).

Anxiety, excessive fears and concerns aren't limited to adult individuals only. Studies report that COVID-19 pandemic has led to the emergence of certain behavioural and emotional problems in children. Some of these problems include increased anxiety levels, excessive fear of family members or loved ones being infected, excessive clinginess and irritability (Imran, Zeshan \& Pervaiz, 2020; Roy et al., 2020). Seniors were also reported to be feeling responsible of the whole family's health and were thus also experiencing anxiety (Kontoangelos, Economou \& Papageorgiou, 2020; Prentice, Zeidan \& Wang, 2020). Children are young and have likely never 
experienced an emergency situation like this. Therefore, limited socialization, shutdown of schools, listening to fear-mongering discussions about COVID-19 from the adults, etc are likely to terrify the child and make them feel distressed. Similarly, seniors are those who are long past their primes - they no longer have the strength or capacity they once possessed. On top of that, COVID-19 is particularly more dangerous to the elderly population. Thus, the elderly population are also particularly sensitive to anxiety in this regard.

Frontline and non-frontline healthcare workers are rightly considered as the heroes and heroines during these tough times. They are the ones who identify and treat the COVID-19 patients. However, researches have reported that there COVID-19 pandemic situation has revealed a significant lack of medical staff in the healthcare sectors (Collado-Boira et al., 2020). Inadequate amount of medical staff had led the government to voluntary recruit medical students into the professional practice fields (Collado-Boira et al., 2020). The prevalence of high anxiety among the healthcare workers have been reported across the literature (Collado-Boira et al., 2020; Dubey et al., 2020; Huang \& Zhao, 2020; Sheraton et al., 2020; Suryavanshi et al., 2020). The two main concerns for their anxiety was working with infected patients and unintentionally transmitting the virus to their family members or loved ones.

As discussed below, researches also report the increased prevalence of anxiety among the psychiatric population. These studies show an increased relapse rate or the worsening of psychiatric conditions in individuals with a history of psychiatric illnesses or currently having one. Failure to follow their psychiatric appointments, being out of medications, having lack of transportations, living in an environment of expression emotions, etc are all related to the worsening of their conditions (Dalal et al., 2020; Gopal, Sharma \& Subramanyam, 2020; Hao et al., 2020; Imran, Zeshan \& Pervaiz, 2020; Muruganandam et al., 2020). Migrant workers are also reported to be having an increased prevalence of anxiety due to several reasons like being fired, having no job security, no adequate pay, having no safe or fixed place to stay, etc. A huge number of suicides have also been reported in this population group.

\section{Depression}

Along with anxiety, the prevalence of depression has also been reported to have increased in each of the aforementioned population groups (Gopal, Sharma and Subramanyam, 2020; Hao et al., 2020; Özdin and Özdin, 2020; Roy et al., 2020; Sheraton et al., 2020; Shuja et al., 2020; Suryavanshi et al., 2020). Social distancing leading to social isolation, disruption of one's daily routines and functioning, inability to go out and have fun, repeated extensions of lockdowns, having no certainty about when the pandemic will be over, having a sedentary lifestyle due to lockdown, having direct (self) or indirect (family members, friends or loved ones) experiences with COVID19 , stuck living or working in an unwanted place or extreme conditions, and the like have been highlighted as the most prominent reasons for the increased prevalence of depression worldwide (Hao et al., 2020; Roy et al., 2020; Sheraton et al., 2020).

\section{Other emotional issues}

After the announcement of COVID-19 and its nature, the masses were reported to panic buy and hoard masks, handwashes, sanitizers and toilet papers (Muthalaly, 2020). Hoarding behaviours were also seen for other household commodities like rice, vegetables and the like. When people are faced with the uncertainty like the current pandemic situation, they want to be prepared and want to have a sense of control of their surroundings (Kolitz, 2020). Hoarding and excessive buying of necessary commodities may help in gaining or maintaining this sense of control.

Aggression, excessive anger, government blaming, and domestic violence have also been reported in the literature (Chaturvedi \& Sharma, 2020; Serlachius, Badawy \& Thabrew, 2020; Shuja et al., 2020). Stigmatization, absence of meaningful social contacts, inability to express or vent emotions through other activities like sports, taking a walk, etc, can be the reasons that lead to frustrations in an individual. Aggression and irritability is considered as externalizing behavioural manifestations of COVID-19 (Mukherjee, Bandopadhyay \& Chatterjee, 2020).

A sedentary lifestyle, unbalanced diet or unhealthy eating patterns are the chief factors behind obesity and body image issues (Gaddad et al., 2020). Due to strict lockdown regulations, individuals are unable to go out to gyms, go out for jogging and exercising, unable to follow the 
diets they were following and maybe sometimes emotional eating (Cherikh et al., 2020). Individuals have reported feeling or perceiving their bodies to have become fatter and this affects their perceptions of their selves and ultimately harms their self-esteem if they perceive themselves to be far away from what they want to be (Matsungo \& Chopera, 2020).

Feelings of loss of control, helplessness and uncertainty about the future has not only made people feel depressed but have also pushed some towards suicide (Poudel \& Subedi, 2020; Roy et al., 2020). Resilience is a phenomenon that refers to the individual's capability to mentally and emotionally cope with a stressful event. Studies have reported individual's resilience to be negatively correlated with anxiety, stress and depression symptoms (Gopal, Sharma \& Subramanyam, 2020) and thus, they have encouraged the authorities and societies as a whole to emphasize, to teach and practice building the resilience in an individual (Dalal et al., 2020; Serlachius, Badawy \& Thabrew, 2020).

\section{Conclusion and Recommendation}

\subsection{Psychological Complications}

\section{Personality Traits}

Researches exploring the relationship between personality traits and COVID-19 report that personality traits are an important indicator of an individual's coping style (Afshar et al., 2015). Among the five factors from the Five Factor Model by Costa and McCrae (McCrae and John, 1992), individuals high in the domains of 'Openness to experience' and 'Conscientiousness' were found to be significantly positively correlating with emotion-oriented and task-oriented coping style, while negatively correlated with avoidance-oriented coping style (Prentice, Zeidan \& Wang, 2020). The explanation behind this is that these individuals are more open to new ideas and learning opportunities, they are flexible to situations, and they see the current pandemic situation as an opportunity for self-development rather than a hindrance or challenge. These individuals are also highly organized and more likely to follow the protective measures and make sure everyone, including themselves, is safe.

\section{Emotional Intelligence}

Emotional Intelligence (EI) is the ability to be aware of and manage the emotions of oneself and that of others. As explained earlier, the emergence and the fast spread of COVID-19 have led to many emotional disturbances in people (Dalal et al., 2020; Sheraton et al., 2020). People with high EI are likely to cope better during these times. Researchers have found EI to be significantly positively correlated with task-oriented and emotion-oriented coping style (Prentice, Zeiden \& Wang, 2020).

\section{Virtual World}

COVID-19 has also compelled the world to turn to virtuality and Information and Communication Technology (ICT) for its functioning. Since face-to-face contacts are deemed a threat to health, educational institutes, workplaces, shops, and restaurants have all been shut down (BBC News, 2020; Business Today, 2020). As a result, the practices of remote working, Work From Home (WFH), and E-learning have emerged (Nyambuya et al., 2021). To cope with and further prevent any more academic and organizational losses, institutes used software like MS Teams, Skype, Zoom, Google Meet, etc., for its functioning. Consequently, there has been a massive increase in students and corporate office workers (Imran, Zeshan \& Pervaiz, 2020; Khattar, Jain \& Quadri, 2020). Studies have shown that the duration of screen-time ranges from 30 minutes to more than 12 hours a day (Khattar, Jain \& Quadri, 2020; Nagata, Magid \& Gabriel, 2020).

While turning to virtuality is a good solution for the time being, it must be considered that not everyone has unlimited access to the internet. Thus, those with connectivity problems miss out on a lot of work or are even being fired (Posel, Oyenubi \& Kollamparambil, 2021; Sneed, 2020). This creates additional social, psychological, and financial stress on the individual and hampers their well-being. Additionally, major concerns with e-learning lie with learning disruptions for the student population groups due to poor network, mode of examination and grading, and admissions to the next level of education (Khattar, Jain \& Quadri, 2020). Therefore, prolonged screen-time creates monotony and frustrations due to poor network; it gives rise to inappropriate 
sleeping and eating habits that are all detrimental to the individual's health and overall well-being (The Economic Times, 2020).

\section{Relationship with self and others}

Lockdowns have also proved to cause some positive changes. Researches report that lockdowns have enabled individuals to connect with their families, neighbours, friends, or relatives and improve their social relationships (Khatter, Jain \& Quadri, 2020). For recreational activities, people have reported engaging in internet gaming, watching movies, spending time with their loved ones, sleeping and relaxing, cooking, learning or mastering a new skill (Desai, 2020; Bhandari, 2020). Lockdowns have also helped individuals to engage in self-care and have provided them with opportunities to introspect.

While learning a skill during lockdown is one of the positive consequences of this lockdown, it also has a negative side. Everyone is learning or doing something new and posting on social media platforms (News18, 2021). This motivates or pressurizes an individual also to do something new. Media demand or expect all people to develop a new skill, but not everyone is capable of doing that or has the wish to do so (News18, 2021) in the first place. However, they are likely to force themselves to stay relevant on social media platforms (Poudel \& Subedi, 2020). This creates a psychological burden and mental stress upon the individuals, which further provokes negative emotions like shame, guilt, and regret.

\subsection{Social Complications}

\section{Occupational Stress}

As mentioned earlier, healthcare workers are the most important figures during the pandemic situation. These individuals continue to work every day, putting themselves at risk under extreme conditions regardless of the fact if they are working with COVID-19 patients or not. This creates tremendous amounts of occupational stress that, coupled with anxiety and depression (as explained earlier), can prove to be detrimental to their well-being (Sheraton et al., 2020). Other instances of occupational stress can be seen in corporate office workers. Turning to virtuality made it so that there is no longer a fixed 9-5 working schedule. Because they are at the comfort of their homes, they are supposed to be available for work 24/7 (Madhavan, 2020). This disrupts the worklife balance and creates unnecessary occupational stress.

\section{Abuse and Neglect}

Not everyone has a happy home. The lifetime prevalence of child abuse in India is alarmingly common (19.9\%) (Kumar, Kar \& Kumar, 2019), while the prevalence of self-reported elder (60+year-olds) abuse is $15.7 \%$ (Nagi et al., 2020). There are instances of dysfunctional families and unhappy homes worldwide. Lockdown has compelled individuals to stay at home with their families and has forbidden individuals to go out. This provokes an increased risk of child and elder abuse or neglect at home (Imran, Zeshan \& Pervaiz, 2020). Non-governmental organizations (NGOs) can help track down these victims and provide them with a safe space during COVID-19.

\section{Stigmatization and Government Blaming}

COVID-19 has also brought along with it the act of stigmatization (Bhanot et al., 2020). There is an increased negative social trend of stigmatization against COVID-19 victims, suspected COVID-19 victims, Healthcare workers who treat COVID-19, and even police personnel (Baldassarre et al., 2020; Bhanot et al., 2020). The term "corona positive" brings with it not only the dreadful health news but also the threat of stigmatization which leads to fear of isolation, fear of being "outcasted", racism, violence, discrimination, and marginalization within all the socioeconomic strata of the society (Bhattacharya, Banerjee \& Rao, 2020; Chaturvedi \& Sharma, 2020; Roya et al., 2020). A sick patient with normal cold and fever or a positively tested COVID-19 patient is looked down upon in society. This leads to people hiding or distorting their identity and test results and being reluctant to seek medical help, leading to the worsening of the COVID-19 pandemic situation (Devakumar et al., 2020; Mukherjee, Bandopadhyay \& Chatterjee, 2020).

In the 'Panic Framework' proposed by Nicomedesa and Avila (2020), it was stated that individuals display a range of patterns of behaviour, feeling, and thinking. Of them all, government blaming is of most relevance here. Government blaming refers to the frustration of its citizens directed 
towards the government due to bad things happening. The spread of COVID-19 was once blamed due to ineffective government actions, and this action is more likely to lead to the defiance of authorities. However, India soon gained international recognition for its most effective way of handling COVID-19 and having a surprisingly "low" amount of COVID-19 cases for its population. Trusting in one's government's actions and believing that it will work leads to compliance and obedience to the authority. India is an example of this successful obedience to authority (Times of India, 2020).

\section{Willingness to Pay for the increased usage of electricity}

As explained earlier, turning to virtuality means an increased screen-time, meaning that devices are on for practically most of the day. This leads to a tremendous increase in the electricity bill. Studies exploring one's willingness to pay the electricity bill and adopting smart technology home reveals individuals were reluctant to adopt the smart technology for their homes (Chen, Rubens \& $\mathrm{Li}, 2020)$. Attitude perceived behavioural control for electrical usage and social norms was the strongest predictor of willingness to pay. Additionally, people were willing to pay more if it included their well-being and if they had moderate to high levels of the perceived risk of being infected by COVID-19 (Chen, Rubens \& Li, 2020).

Unable to socialize has also led to worsening of one's social skills and have, thus, impacted one's social development (Chaturdevi \& Sharma, 2020). Absence of non-verbal communication renders an individual to solely rely on the word phrasing to figure out how the other person is thinking or feeling. Non-verbal cues form an important part of communication. The absence of them can make an individual misinterpret or misunderstand the message's meaning, thus creating confusion, dilemma, or even interpersonal conflict. Additionally, since handshakes are forbidden or cannot be done in these COVID-19 times, studies have proposed to replace handshakes with Indian greeting styles of "Namaste" (Chaturvedi \& Sharma, 2020).

\section{Future Scope and Limitations of this Study}

\subsection{Limitations}

This study was done with a literature review of only 30 research articles and research papers. Thus, the findings from this paper may not apply for generalization to the masses. Additionally, this paper did not explore the mental health effects of the marginalized population groups, i.e. the prisoners, the immigrants, and the slum dwellers.

\subsection{Recommendations (Future Scope)}

In times of COVID-19, when the world is halted yet time is ever so precious for families, students, office workers, healthcare workers, and the like, the following steps can be taken at an individual and collective level:

a) Self-care: Individuals are encouraged to engage in self-care activities. These activities do not necessarily always have to be learning a new skill. Self-care activities can also be taking care of one's hair and skin, sleeping, stargazing, etc.

b) Practicing acceptance: COVID-19 is a pandemic that has already arrived and is here to stay for a while. Accepting the reality will better facilitate an individual to adjust to the situation, regain their sense of control and plan accordingly for their future.

c) Being aware: These rules and regulations taken as protective measures against COVID-19 exist for a reason. Individuals are encouraged to read-only necessary information about the pandemic situation (e.g., its nature, origin, risk factors, cure progression, and infectivity/mortality rates). Media have been reported to exaggerate the pandemic situation by a whole lot. Thus, following every little update posted on social media will not be helpful and will only be detrimental to one's inner peace.

d) Reconnecting: Since face-to-face contact is not an available option at the moment, individuals are encouraged to try reconnecting with their family members, their friends or relative, their neighbours, their pets, and even with their favourite books via digital methods.

e) Maintaining a log or journal: Individuals are encouraged to maintain a journal or a log weekly, daily or monthly (however they like). This would allow them to track their moments, activities, 
feelings and thoughts, and help reminisce or hope for a better future - a future when one day they would be looking back at these logs and feeling a sense of accomplishment because they made it through.

Future researchers are encouraged to explore the mental health effects of COVID-19 on the marginalized population (e.g. the prisoners, the immigrants, and the slum dwellers) more to have a comprehensive and holistic understanding of the people who have been overshadowed by the society yet form just an important part of it. In addition, longitudinal studies are also encouraged to be done to understand and compare the pre-COVID19 and post-COVID19 scenarios to gain a comprehensive picture of the mental health effects of COVID-19.

\section{Conclusion}

The findings of this paper suggest that the mental health effects of COVID-19 are not always visible to or thought of as a priority in these dire times of extreme social distancing and lockdown measures. Strict lockdown laws, social distancing, restrictions in movement, and increased screen time have been reported to disrupt one's daily or mainstream functioning and, thus, jeopardize their mental health and overall well-being. In addition, constant misinformation in social media portals is also responsible for causing panic and anxiety, and depression, which sometimes leads to suicidal ideation or suicide. These effects are not restricted to the general population; they also affect other population groups such as children and older adults, individuals with special needs, the frontline and non-front-line healthcare workers, migrants, wage workers, and individuals with pre-existing psychiatric illnesses. However, it has been reported that some of these population groups are not given much of a priority. Thus, there is an urgent need for mental health intervention plans and strategies that can help alleviate the (excessive) psychological distress related to COVID-19 is putting on all of the population above groups. Certain recommendations and limitations of this study are also given.

\section{References}

Afshar, H., Roohafza, H. R., Keshteli, A. H., Mazaheri, M., Feizi, A., \& Adibi, P. (2015). The association of personality traits and coping styles according to stress level. Journal of research in medical sciences: the official journal of Isfahan University of Medical Sciences, 20(4), 353-358.

Baldassarre, A., Giorgi, G., Alessio, F., Lulli, L. G., Arcangeli, G., \& Mucci, N. (2020). Stigma and discrimination (SAD) at the time of the SARS-CoV-2 pandemic. International Journal of Environmental Research and Public Health, 17(17), 6341-6364.

BBC News. (2020, May 17). India extends coronavirus lockdown by two weeks. BBC News. Retrieved from: https://www.bbc.com/news/world-asia-india-52698828.

Bhandari, K. (2020, May 14). Lockdown bringing couples closer, says new survey. The Hindustan Times. Retrieved from: https://www.hindustantimes.com/sex-and-relationships/lockdown-bringingcouples-closer-says-new-survey/story-UyIRsffEmGEzoCI0HRzbXL.html.

Bhanot, D., Singh, T., Verma, S. K., \& Sharad, S. (2020). Stigma and discrimination during COVID-19 pandemic. Frontiers in public health, 8, 829.

Bhattacharya, P., Banerjee, D., \& Rao, T. S. (2020). The "Untold" Side of COVID-19: Social Stigma and Its Consequences in India. Indian Journal of Psychological Medicine, 42(4), 382-386.

Business Today. (2020, April 15). Lockdown 2.0: Restaurants, bars shut but dhabas on highways to remain open. Business Today. Retrieved from: https://www.businesstoday.in/current/corporate/lockdown-20-restaurants-bars-shut-but-dhabason-highways-to-remain-open/story/401058.html.

Chaturvedi, S. K., \& Sharma, M. K. (2020). Psychosocial aspects of Covid-19, the Indian way. World Social Psychiatry, 2(2), 129-131.

Chen, C. F., de Rubens, G. Z., Xu, X., \& Li, J. (2020). Coronavirus comes home? Energy use, home energy management, and the social-psychological factors of COVID-19. Energy research \& social science, 68, 101688.

Cherikh, F., Frey, S., Bel, C., Attanasi, G., Alifano, M., \& Iannelli, A. (2020). Behavioral food addiction during lockdown: time for awareness, time to prepare the aftermath. Obesity surgery, 30, 35853587.

Collado-Boira, E. J., Ruiz-Palomino, E., Salas-Media, P., Folch-Ayora, A., Muriach, M., \& Baliño, P. (2020). "The COVID-19 outbreak"-An empirical phenomenological study on perceptions and psychosocial considerations surrounding the immediate incorporation of final-year Spanish nursing 
and medical students into the health system. Nurse Education Today, 104504.

Dalal, P. K., Roy, D., Choudhary, P., Kar, S. K., \& Tripathi, A. (2020). Emerging mental health issues during the COVID-19 pandemic: An Indian perspective. Indian Journal of Psychiatry, 62(9), 354364.

Desai, S. (2020, May 14). How the lockdown is cementing relationships and bringing families together. Times of India. Retrieved from: https://timesofindia.indiatimes.com/life-style/spotlight/how-thelockdown-is-cementing-relationships-and-bringing-families-together/articleshow/75731732.cms.

Devakumar, D., Shannon, G., Bhopal, S. S., \& Abubakar, I. (2020). Racism and discrimination in COVID-19 responses. The Lancet, 395(10231), 1194-1194.

Dubey, S., Biswas, P., Ghosh, R., Chatterjee, S., Dubey, M. J., Chatterjee, S., ... \& Lavie, C. J. (2020). Psychosocial impact of COVID-19. Diabetes \& Metabolic Syndrome: Clinical Research \& Reviews.

Ferenhof, H. A., \& Fernandes, R. F. (2016). Desmistificando a revisão de literatura como base para redação científica: método SSF. Revista $A C B, 21(3), 550-563$.

Gaddad, P., Pemde, H. K., Basu, S., Dhankar, M., \& Rajendran, S. (2018). Relationship of physical activity with body image, self-esteem sedentary lifestyle, body mass index and eating attitude in adolescents: A cross-sectional observational study. Journal of family medicine and primary care, 7(4), 775-779.

Gopal, A., Sharma, A. J., \& Subramanyam, M. A. (2020). Dynamics of psychological responses to COVID-19 in India: A longitudinal study. PloS one, 15(10), e0240650, 1-15.

Grover, S., Sahoo, S., Mehra, A., Avasthi, A., Tripathi, A., Subramanyan, A., ... \& Chakraborty, K. (2020). Psychological impact of COVID-19 lockdown: An online survey from India. Indian Journal of Psychiatry, 62(4), 354.

Guo, Y. R., Cao, Q. D., Hong, Z. S., Tan, Y. Y., Chen, S. D., Jin, H. J., ... \& Yan, Y. (2020). The origin, transmission and clinical therapies on coronavirus disease 2019 (COVID-19) outbreak-an update on the status. Military Medical Research, 7(1), 1-10.

Hamza Shuja, K., Aqeel, M., Jaffar, A., \& Ahmed, A. (2020). COVID-19 pandemic and impending global mental health implications. Psychiatria Danubina, 32(1), 32-35.

Hao, F., Tan, W., Jiang, L., Zhang, L., Zhao, X., Zou, Y., ... \& Tran, B. (2020). Do psychiatric patients experience more psychiatric symptoms during COVID-19 pandemic and lockdown? A case-control study with service and research implications for immunopsychiatry. Brain, behavior, and immunity.

Hebbar, N. (2020, March 24). Coronavirus | PM's address to the nation updates: lockdown extended to entire country for next 21 days, says Modi. The Hindu. Retrieved from: https://www.thehindu.com/news/national/prime-minister-narendra-modi-live-updates-march-242020/article31153585.ece.

Hossain, M. M., Purohit, N., Sharma, R., Bhattacharya, S., McKyer, E. L. J., \& Ma, P. (2020). Suicide of a farmer amid COVID-19 in India: Perspectives on social determinants of suicidal behavior and prevention strategies.

Huang, Y., \& Zhao, N. (2020). Generalized anxiety disorder, depressive symptoms and sleep quality during COVID-19 outbreak in China: a web-based cross-sectional survey. Psychiatry research, 112954.

Imran, N., Zeshan, M., \& Pervaiz, Z. (2020). Mental health considerations for children \& adolescents in COVID-19 Pandemic. Pakistan Journal of Medical Sciences, 36(COVID19-S4), 67-72.

Keyes, C. L. (2014). Bridging Occupational, Organizational and Public Health.

Khattar, A., Jain, P. R., \& Quadri, S. M. K. (2020, May). Effects of the disastrous pandemic COVID 19 on learning styles, activities and mental health of young Indian students-a machine learning approach. In 2020 4th International Conference on Intelligent Computing and Control Systems (ICICCS) (pp. 1190-1195). IEEE.

Kochhar, A. S., Bhasin, R., Kochhar, G. K., Dadlani, H., Mehta, V. V., Kaur, R., \& Bhasin, C. K. (2020). Lockdown of 1.3 billion people in India during Covid-19 pandemic: A survey of its impact on mental health. Asian journal of psychiatry, 54, 102213.

Kolitz, D. (2020, April 5). Why Does Spending Money Feel So Good? Retrieved from: https://gizmodo.com/why-does-spending-money-feel-so-good-1842593770.

Kontoangelos, K., Economou, M., \& Papageorgiou, C. (2020). Mental health effects of COVID-19 pandemia: a review of clinical and psychological traits. Psychiatry investigation, 17(6), 491-505.

Kumar, M. T., Kar, N., \& Kumar, S. (2019). Prevalence of child abuse in Kerala, India: An ICAST-CH based survey. Child Abuse \& Neglect, 89, 87-98.

Lamers, S. M., Westerhof, G. J., Bohlmeijer, E. T., ten Klooster, P. M., \& Keyes, C. L. (2011). Evaluating 
the psychometric properties of the mental health continuum-short form (MHC-SF). Journal of clinical psychology, 67(1), 99-110.

Madhavan, A. (2020, June 11). Overworked @home: New norm of work from home turning out to be a taxing one for employees The New Indian Express. Retrieved from https://www.newindianexpress.com/cities/chennai/2020/jun/11/overworked-home-2154904.html.

Majumdar, P., Biswas, A., \& Sahu, S. (2020). COVID-19 pandemic and lockdown: cause of sleep disruption, depression, somatic pain, and increased screen exposure of office workers and students of India. Chronobiology International, 37(8), 1191-1200.

Manjunath, B.S. (2020, April, 14). Covid-19: 8 ways in which technology helps pandemic management. The Economic Times. Retrieved from https://cio.economictimes.indiatimes.com/news/next-gentechnologies/covid-19-8-ways-in-which-technology-helps-pandemic-manag.

Matsungo, T. M., \& Chopera, P. (2020). The effect of the COVID-19 induced lockdown on nutrition, health and lifestyle patterns among adults in Zimbabwe. medRxiv.

McCrae, R. R., \& John, O. P. (1992). An introduction to the five-factor model and its applications. Journal of personality, 60(2), 175-215.

Moghanibashi-Mansourieh, A. (2020). Assessing the anxiety level of Iranian general population during COVID-19 outbreak. Asian journal of psychiatry, 102076.

Mukherjee, A., Bandopadhyay, G., \& Chatterjee, S. S. (2020). COVID-19 pandemic: mental health and beyond-The Indian perspective. Irish Journal of Psychological Medicine, 38, 140-144.

Muruganandam, P., Neelamegam, S., Menon, V., Alexander, J., \& Chaturvedi, S. K. (2020). COVID-19 and Severe Mental Illness: Impact on patients and its relation with their awareness about COVID19. Psychiatry Research, 291, 113265.

Muthalaly, S. (2020, April 15). The Stock-home syndrome: How to deal with panic buying during the COVID-19 pandemic. The Hindu. Retrieved from: https://www.thehindu.com/society/the-stockhome-syndrome-how-to-deal-with-panic-buying-during-the-covid-19.

Nagata, J. M., Abdel Magid, H. S., \& Pettee Gabriel, K. (2020). Screen time for children and adolescents during the coronavirus disease 2019 pandemic. Obesity, 28(9), 1582-1583.

Nagi, R. S., Sembiah, S., Chatterjee, A., \& Negi, R. (2020). What is the Status of Elderly Abuse in India: A Systematic Review Protocol. medRxiv.

News18. (2021, April 29). Between Hope and Despair: Social Media is the Band-Aid Keeping India Together amid Covid-19. News18. Retrieved from: https://www.news18.com/news/buzz/betweenhope-and-despair-social-media-is-the-band-aid-thats-keeping-india-together-amid-covid-193687860.html.

Nicomedes, C. J. C., \& Avila, R. M. A. (2020). An analysis on the panic during COVID-19 pandemic through an online form. Journal of affective disorders, 276, 14-22.

Nyambuya, V.P., Nyamaruze, P., Dube, M., \& Shumba, K. (2021). Rethinking education in the age of 'social distancing': A qualitative inquiry on University of KwaZulu-Natal students' responses to online learning in the context of COVID-19. African Journal of Education, 2 (1), 105-124

Özdin, S., \& Bayrak Özdin, S. (2020). Levels and predictors of anxiety, depression and health anxiety during COVID-19 pandemic in Turkish society: The importance of gender. International Journal of Social Psychiatry, 504-511.

Posel, D., Oyenubi, A., \& Kollamparambil, U. (2021). Job loss and mental health during the COVID-19 lockdown: Evidence from South Africa. PloS one, 16(3), e0249352.

Prentice, C., Zeidan, S., \& Wang, X. (2020). Personality, trait EI and coping with COVID 19 measures. International Journal of Disaster Risk Reduction, 51, 101789.

Rajkumar, R. P. (2020). COVID-19 and mental health: A review of the existing literature. Asian journal of psychiatry, AJP 102066.

Roy, A., Singh, A. K., Mishra, S., Chinnadurai, A., Mitra, A., \& Bakshi, O. (2020). Mental health implications of COVID-19 pandemic and its response in India. The International journal of social psychiatry. 1-14.

Roy, D., Tripathy, S., Kar, S. K., Sharma, N., Verma, S. K., \& Kaushal, V. (2020). Study of knowledge, attitude, anxiety \& perceived mental healthcare need in Indian population during COVID-19 pandemic. Asian Journal of Psychiatry, 102083.

Serlachius, A., Badawy, S. M., \& Thabrew, H. (2020). Psychosocial Challenges and Opportunities for Youth With Chronic Health Conditions During the COVID-19 Pandemic. JMIR Pediatrics and Parenting, 3(2), e23057.

Sheraton, M., Deo, N., Dutt, T., Surani, S., Hall-Flavin, D., \& Kashyap, R. (2020). Psychological effects of the COVID 19 pandemic on healthcare workers globally: A systematic review. Psychiatry 
research, 292, 113360.

Skirbekk, V., \& James, K. S. (2014). Abuse against elderly in India-The role of education. BMC public health, 14(1), 336-344.

Sneed, T. (2020). The Effect of COVID-19 on Law Libraries: Are These Changes Temporary or a Sign of the Future?. Washburn LJ, 60, 107-129.

Suryavanshi, N., Kadam, A., Dhumal, G., Nimkar, S., Mave, V., Gupta, A., ... \& Gupte, N. (2020). Mental health and quality of life among healthcare professionals during the COVID-19 pandemic in India. Brain and behavior, 10(11), 1-12.

The Economic Times. (2020, October 24). Covid resulted in poor sleep quality, bad eating habits and anxiety. The Economic Time. Retrieved https://economictimes.indiatimes.com/magazines/panache/covid-resulted-in-poor-sleep-qualitybad-eating-habits-and-anxiety/articleshow/78845071.cms?from=mdr.

Times of India. (2020, May 11). Commendable that India has been able to keep Covid-19 cases very low compared to other nations: WHO's chief scientist. The Times of India. Retrieved from: https://timesofindia.indiatimes.com/india/commendable-that-india-has-been-able-to-keep-covid19-cases-very-low-compared-to-other-nations-whos-chief-scientist/articleshow/75675691.cms.

Wardell, J. D., Kempe, T., Rapinda, K. K., Single, A., Bilevicius, E., Frohlich, J. R., ... \& Keough, M. T. (2020). Drinking to Cope During COVID-19 Pandemic: The Role of External and Internal Factors in Coping Motive Pathways to Alcohol Use, Solitary Drinking, and Alcohol Problems. Alcoholism: Clinical and Experimental Research, 44(10), 2073-2083. 